\title{
CASE REPORT \\ Ultrasonographically guided obturator nerve block for bilateral adductor spasticity in a paraplegic patient
}

\author{
E Yalcin, M Akyuz, O Ilgu and NB Ozer
}

\begin{abstract}
Study design: A case report defining an interventional procedure.
Objectives: To reveal the role of the ultrasonography in nerve blocks at difficult anatomical locations of the obturator nerve.

Setting: The study was conducted in Ankara, Turkey.

Methods: A paraplegic patient with lower limb adductor spasticity treated with phenol block using the guidance of ultrasonography. Results: After treatment of the adductor spasticity by obturator nerve block the patient achieved an upright standing position with a walker. Hygienic care was improved and spasticity-related pain was reduced.
\end{abstract}

Conclusion: Ultrasound is a challenging tool for nerve blocks. Ultrasound guidance seems as an essential approach for the blocking of the nerves with variable anatomical courses, such as the obturator nerve.

Spinal Cord (2014) 52, S24-S26; doi:10.1038/sc.2014.97

\section{INTRODUCTION}

An obturator block is commonly used for management of adductor spasticity. Complications are rare; however, an obturator block is technically more difficult than other nerve blocks due to the complicated anatomy challenges and the huge variability of the obturator nerve. The obturator nerve is located in the pelvis through obturator foramen and divides into superficial and deep branches. There are anatomical variances of the obturator nerve divisions such as in $50 \%$ of individuals the common obturator nerve divides into superficial and deep branches at the level of the obturator foramen, whereas the others have a multiple branching pattern through the area. It is found that the obturator nerve in the anterior and posterior branches was intrapelvic $(23.22 \%)$, within the obturator canal $(51.78 \%)$ or in the thigh $(25 \%)$. Also high failure rate is reported owing to the deep location of obturator nerve. These complicated anatomies of the obturator nerve could explain the frequent difficulty encountered in the application of obturator nerve blocks. Technically, achieving an optimal position of the patient to perform the obturator nerve block is also difficult. In some cases it is difficult to rotate and abduct the leg due to the spasticity of the lower limb.

Owing to these technical difficulties, many clinicians seem to be reluctant to perform obturator blocks. ${ }^{1-3}$ In this report we have aimed to emphasize the essential role of ultrasound in an obturator block procedure.

\section{CASE REPORT}

A 29-year-old man with a neurological status of T5 American Spinal Injury Association impairment scale A was presented to our rehabilitation center with bilateral severe adductor spasticity. It was difficult to apply the regular rehabilitation program due to the severe spasticity. Adductor spasticity complicated bladder and bowel care for the caregivers. He had taken baclofen $70 \mathrm{mg}$ per day for 6 months but there was no perceived improvement in spasticity. Evaluations were carried out just before the phenol injection was made and also on day 2, 4 weeks and 6 months after the injection. On pretreatment, the Numerical Rating Scale (NRS) for pain intensity was 7, the Modified Ashworth Scale (MAS) scores for the adductors were a $4 / 4$ and the passive range of motion (ROM) in abduction in both of the hips was $30^{\circ}$. After obtaining written consent, neurolysis using $5 \%$ phenol was applied bilaterally with the guidance of ultrasonography along with peripheral nerve stimulator guidance. First, on the right side a high-frequency probe $(12 \mathrm{MHz})$ had been placed just distal to the inguinal ligament and medial to the femoral artery. Just medial to the femoral vein, pectineus, adductor longus and brevis muscles could be identified. The anterior branch was seen as a hypoechoic spindle in hyperechoic thick fascia between the adductor longus and brevis. The needle was inserted through the midpoint of the probe using an outplane approach. The tip of the needle was seen just near the anterior branch (Figure 1). At the same time, the nerve was stimulated using an initially stimulating current of $5 \mathrm{~mA}(2 \mathrm{~Hz}, 0.1 \mathrm{~ms})$ gradually decreasing to $1 \mathrm{~mA}$. After the contraction was observed, a neurolytic block, $5 \mathrm{ml}$ phenol injection, was applied. Later, posterior branch was also visualized between the adductor brevis and magnus and a 5-ml phenol injection was inserted (Figure 2). Subsequently, nerve block of the left obturator nerve was performed through the same procedure.

A drastic increase in ROM values was observed in hip abduction (Figure 3). After the injection, the NRS was 2, the MAS scores at adductors were a $2 / 4$ and passive ROM in abduction of both hips was $80^{\circ}$. In the long-term follow-up no sensory symptoms developed. Also there has been no documented complication with the use of phenol at the patient discharge (4 weeks after the injection) and at routine follow-up (6 months after the injection). 


\section{DISCUSSION}

The efficacious and satisfactory treatment of adductor spasticity improves the ability to walk, hygienic care and reduces spasticityrelated pain. ${ }^{1,2}$ In this case, initially after the injection a dramatic transformation from bedridden patient to being able to be in an upright standing position with the assistance of a walker was achieved.

Over the past few decades, ultrasonography has gained much more importance in interventional procedures. Ultrasound allows one to use one of the most powerful senses, vision. It is known that vision is the most compelling sense. Imaging techniques like fluoroscopy and computed tomography both could guide the nerve block procedures by providing a visual image of the nerves. But ultrasonography is superior among these modalities as there is no radiation exposure, simplicity in practice, portability and economy. ${ }^{2,4}$

Ultrasound enables the identification of nerves and visualization of the needle up to the final target. The deep localization and huge variability of the obturator nerve could make the block procedure extremely challenging. However, with the assistance of ultrasonography both the time period for performing the block procedure and the number of the needle punctures are significantly reduced. ${ }^{4,5}$

In our case, we used nerve stimulation in addition to ultrasound guidance. This could offer a controversial question 'do we need to use nerve stimulation in addition to ultrasound?' It is not a necessity but nerve stimulation gives us additional information about the physiology and continuity of the nerves. ${ }^{2,5}$ This could help us to assess our expectations from therapy. Some nerves have more certain and easy anatomical approaches, such as suprascapular nerve. Nerve stimulation as an adjunct to ultrasound guidance may have a limited role during these type of operations to this kind of nerve. On the other hand, in nerves with complicated anatomy due to the

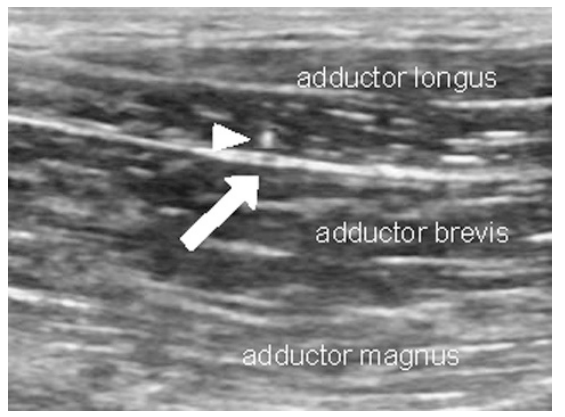

Figure 1 Ultrasonographic image showing the tip of the needle just near the anterior branch of the right obturator nerve. variability of divisions, nerve stimulation could provide a better and more effective block procedure.

Localized treatments of spasticity such as nerve blocks provide the advantage of reducing the harmful spasticity in one area while preserving useful spasticity in another area. ${ }^{6}$ Also in our patient after the spasticity of adductor muscles has been reduced, the spasticity of the other lower extremity muscles like soleus and gastrocnemius help him to maintain an upright position by use of a walker. We considered that after the failure of the baclofen therapy, a local anti-spastic agent would be a better solution compared with offering an alternative oral medication.

In the Botulinum toxin era, the popularity of phenol has been eclipsed. Botulinum toxin is easier in practice and has fewer side effects than phenol injections. On the other hand, phenol is cheap and has a long-lasting effect. Owing to its easy application and lower incidence of complication, the Botulinum toxin seems to be the favorite among many clinicians. ${ }^{1,4}$ But in some cases like overweight patients or patients with multiple spastic muscles or patients with bilateral adductor spasticity, Botulinum toxin would be a limited solution due to insufficient dosage. Compared with Botulinum toxin, phenol blocks have a much more long-term effect. Under these conditions, the phenol injection of the obturator nerve is a good choice for adductor spasticity in spinal cord injury. As in our case, ultrasound challenges to overcome the difficulty in administration of phenol to obturator nerve.

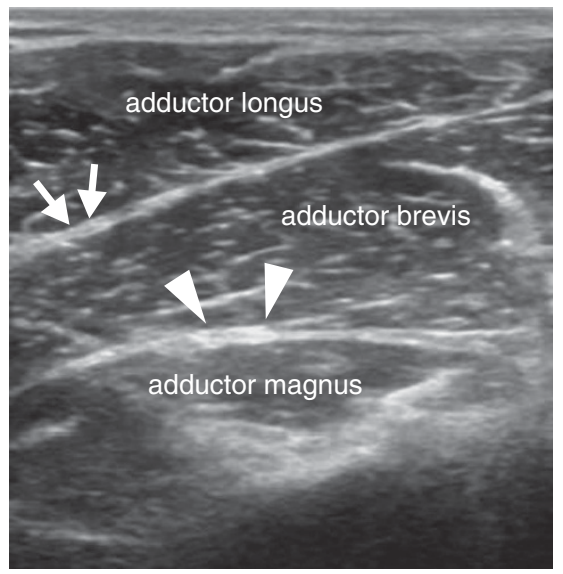

Figure 2 Arrows demonstrate the anterior and arrowheads demonstrate the posterior branches of the right obturator nerve between the adductor muscles.

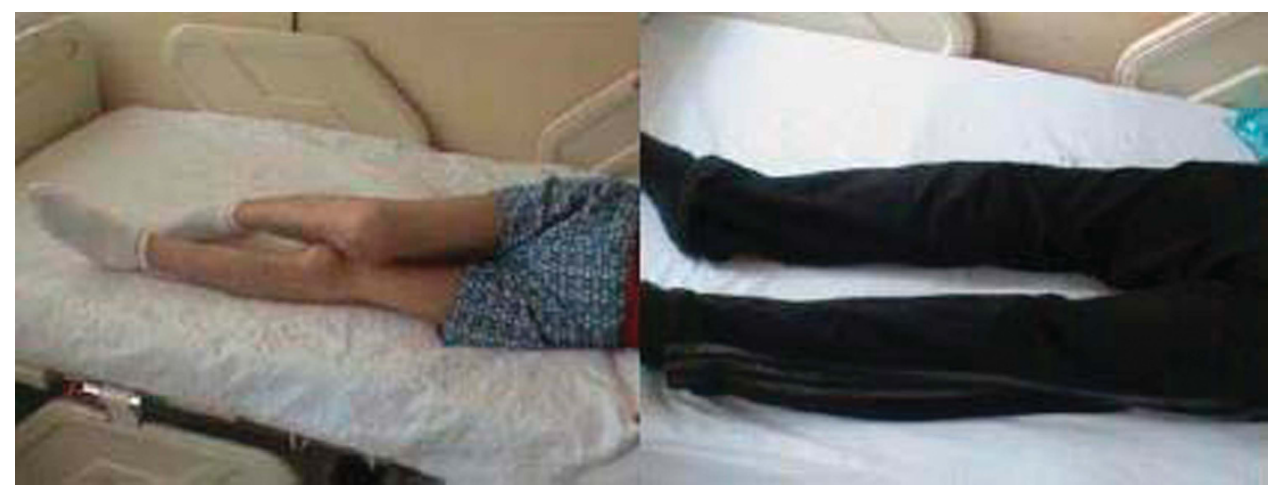

Figure 3 The position of patient before and after the phenol injection. 


\section{CONCLUSION}

Ultrasound is an appropriate imaging modality for nerve blocks that actualizes that 'Seeing is believing. Seeing makes the interventional procedures like nerve blocks more simple and beneficial. The benefits of direct visualization of the nerves, surrounding structures, observation of the needle tip and spread of the local anesthetic make ultrasound-guided nerve injections highly valuable.

\section{CONFLICT OF INTEREST}

The authors declare no conflict of interest.
1 Akkaya T, Unlu E, Alptekin A, Gumus HI, Umay E, Cakci A. Neurolytic phenol blockade of the obturator nerve for severe adductor spasticity. Acta Anaesthesiol Scand 2010, 54: 79-85.

2 Soong J, Schafhalter-Zoppoth I, Gray AT. Sonographic imaging of the obturator nerve for a regional block. Reg Anesth Pain Med 2007; 32: 146-151.

3 Anagnostopoulou S1, Kostopanagiotou G, Paraskeuopoulos T, Chantzi C, Lolis E, Saranteas T. Anatomic variations of the obturator nerve in the inguinal region: implications in conventional and ultrasound regional anesthesia techniques. Reg Anesth Pain Med 2009; 34: 33-39.

4 Cauvin ER. Musculoskeletal ultrasonography: seeing is believing. Vet J 1999; 158: 4 .

5 Denny NM, Griffiths WH. Location, location, location! Ultrasound imaging in regional anaesthesia. Br J Anaesth 2005; 94: 1-2.

6 Kirazli Y, On AY, Kismali B, Aksit R. Comparison of phenol block and botulinus toxin type $\mathrm{A}$ in the treatment of spastic foot after stroke: a randomized, double-blind trial. Am J Phys Med Rehabil 1998; 77: 510-515. 